\title{
Construction of polycythemia vera protein interaction network and prediction of related biological functions
}

\author{
L.-J. Liu', X.-J. Cao' ${ }^{2}$, C. Zhou' ${ }^{1}$, Y. Sun ${ }^{3}$, Q.-L. Lv³, F.-B. Feng ${ }^{1}$, Y.-Y. Zhang ${ }^{4}$ and \\ C.-G. Sun ${ }^{1}$
}

${ }^{1}$ Cancer Center, Weifang Traditional Chinese Medicine Hospital, Kuiwen District, Weifang, Shandong Province, China

${ }^{2}$ The First Clinical College, Shandong University of Chinese Medicine, Changqing District, Jinan, Shandong Province, China

${ }^{3}$ Clinical Medical School, Weifang Medical University, Kuiwen District, Weifang, Shandong Province, China

${ }^{4}$ China Academy of Traditional Chinese Medicine, Dongcheng District, Beijing, China

Corresponding author: C.-G. Sun

E-mail: hxdhxd19852003@163.com

Genet. Mol. Res. 15 (1): gmr.15017169

Received August 8, 2015

Accepted October 15, 2015

Published January 26, 2016

DOI http://dx.doi.org/10.4238/gmr.15017169

ABSTRACT. Here, polycythemia vera (PV)-related genes were screened by the Online Mendelian Inheritance in Man (OMIM), and literature pertaining to the identified genes was extracted and a protein-protein interaction network was constructed using various Cytoscape plugins. Various molecular complexes were detected using the Clustervize plugin and a gene ontologyenrichment analysis of the biological pathways, molecular functions, and cellular components of the selected molecular complexes were identified using the BiNGo plugin. Fifty-four PV-related genes were identified in OMIM. The protein-protein interaction network contains 5 molecular complexes with correlation integral values $>4$. These complexes regulated various biological processes (peptide tyrosinase acidification, cell metabolism, and 
macromolecular biosynthesis), molecular functions (kinase activity, receptor binding, and cytokine activity), and the cellular components were mainly concentrated in the nucleus, intracellular membrane-bounded organelles, and extracellular region. These complexes were associated with the JAKSTAT signal transduction pathway, neurotrophic factor signaling pathway, and Wnt signaling pathway, which were correlated with chronic myeloid leukemia and acute myeloid leukemia.

Key words: Protein-protein interaction network; Polycythemia vera; Online Mendelian Inheritance in Man; Molecular complexes; Biological pathways

\section{INTRODUCTION}

Polycythemia vera (PV) is a chronic and cloned myeloproliferative disease with predominant erythrocytosis and abnormal erythrocyte, granulocyte, and megakaryocyte proliferation. The clinical features of PV include erythrocytosis, neutrophilic leukocytosis, thrombocytosis, and splenomegaly; however, until the latter stages of disease, PV patients exhibit progressive myelofibrosis, anemia, and up-regulation of splenomegaly, symptoms that are also observed in idiopathic myelofibrosis syndrome.

A number of researchers have attempted to elucidate the pathogenesis of $\mathrm{PV}$, with variable degrees of success, since 2005. Mutations in the JAK2 gene have been specifically identified in PV patients by multiple research groups, and is currently considered the standard for PV pathogenesis (Levine and Gilliland, 2008). Additionally, approximately $40 \%$ of the PV patients are believed to express an abnormal chromosomal karyotype. Kralovics et al. (2002) reported that 9pLOH was the most common abnormal chromosome in PV patients, accounting for approximately $33 \%$ of the total. However, these chromosomal abnormalities are also known to affect chronic myeloid leukemia; therefore, these cannot be classified as PV-specific abnormalities (Kralovics et al., 2002). The discovery of mutations in the JAK2 gene is believed to have opened new avenues for research into the pathogenesis of PV; however, as this mutation is also observed in other myeloproliferative disorders, and is not universal to all PV patients, research into PV pathogenesis must not be limited to the JAK2 mutation. Recent advances in the field of bioinformatics have led to the development of new tools (e.g., for the construction of a protein-protein interaction network) to elucidate the genesis and development of PV.

Here, previously confirmed PV-related genes were identified by Online Mendelian Inheritance in Man (OMIM) and a literature search was performed using the Cytoscape and Agilent Literature Search plugin; the obtained information was used to build a protein-protein interaction network. The MCOMD algorithm, obtained from the Clusterviz plugin, was used to identify possible molecular complexes in the network, and the Bingo plugin was used for gene ontology (GO)-enrichment analysis of the biological processes, molecular functions, and cellular components of the complex. The DAVID program was used to enrich the biological pathways of molecular complexes, which in turn provides a basis for further research into the genesis and development of PV.

\section{MATERIAL AND METHODS}

\section{Materials}

PV-related genes were extracted from the OMIM database, using the search term "Polycythemia vera" in the "Gene" section (Amberger et al., 2009; Burkard et al., 2010). 


\section{Methods}

\section{Construction of gene/protein interaction network}

The identified PV-related genes were searched using the Agilent Literature Search 2.7.7 plugin (Cytoscape 2.8.2), which performed a comprehensive literature search of the related literature in PubMed (Vailaya et al., 2005); the retrieved results were read and all false-positive interaction information was excluded. The identified (included) gene/protein interaction relationships were visualized in the form of a network in Cytoscape 2.8.2 (Shannon et al., 2003).

\section{Network analysis}

The MCOMD algorithm in the Clusterviz 1.2 plugin of Cytoscape 2.8.2 was used to perform a regional correlation analysis of the biological network. This analysis provided the integral correlation values and helped identify possible protein groups forming molecular complexes in the entire network. Molecular complexes were annotated as areas with correlation integral values greater than 4 .

The BiNGO 2.44 network analysis plugin of Cytoscape 2.8.2 was used to annotate and enrich the function of each gene/protein in the molecular complexes related to the biological process, molecular function, and cellular components. The "Hypergenometric test" was performed, with $\mathrm{P}<0.05$ as the significant threshold (http://apps.cytoscape.org/apps/bingo).

The names of genes/proteins included in the molecular complexes were submitted to "The Database for Annotation, Visualization, and Integrated Discovery" (Dennis Jr. et al., 2003; Huang et al., 2009); a relative search of the KEGG, BBID, PANTHER, PATHWAY, BIOCARTA, EC_NUMBER, and REACTOME_PATHWAY databases helped identify the biological pathways regulated by $\mathrm{PV}$-related genes. The $\mathrm{P}$ values of relative target genes predicted using the software were calculated by the incidental Fisher exact test. $P<0.05$ indicated the role of these genes in transduction pathways with statistical significance.

\section{Major outcome measures}

The nodes (proteins) and sides (interaction) of a protein-protein interaction network comprised of PV-related genes in the molecular complexes, and the correlation integral values were determined. The biological processes regulated by molecular functions, cellular components, and biological pathways of these molecular complexes were identified.

\section{RESULTS}

\section{PV-related genes in OMIM}

Fifty-six PV-related genes were identified using the search criterion (up to 2015-02-09); fifty-four genes (up to 2015-02-09) were retained after screening and removal of duplicate genes: CTNNB1, ACSL6, ASXL1, BCL2L1, BCR, CALR, CD177, CRP, CSF2, CTNNB1, DKK3, DNMT3A, EIF2S1, ERCC2, ETV6, F2, F5, FIP1L1, HAMP, GSTT1, HDAC11, HDAC9, HGF, HMGA2, IDH1, IDH2, IFNA1, IL11, IL23A, JAK2, KIT, L3MBTL1, LCN2, MEFV, MPL, MTHFR, NFE2, NFIX, NR3C1, PDGFRA, PLVAP, PRM2, PTX3, SH2B3, SOCS2, SOCS3, SPRY1, SPRY2, SPRY4, STAT3, STAT5A, TET2, TNFRSF11B, and TNF. 


\section{Gene/protein interaction network}

A gene/protein interaction network, consisting of 541 nodes (protein) and 1771 sides (interactions), was constructed after a comprehensive literature search of the 54-related genes using the Agilent Literature Search engine (Figure 1). The yellow color in Figure 1 indicates genetic diseaserelated proteins in OMIM, while the red color indicates the proteins identified by a literature search.
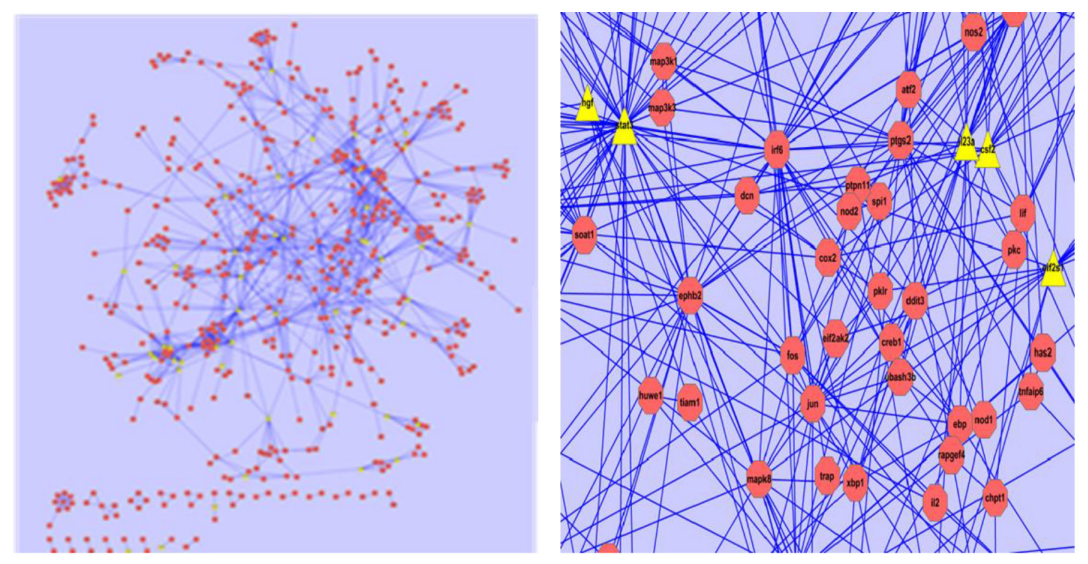

Figure 1. Protein-protein interaction network of polycythemia vera (the whole and the part).

\section{Molecular complex}

The MCOMD algorithm was used to analyze the interaction network; five molecular complexes with correlation integral values higher than 4 (Figure 2) were identified. The yellow color in Figure 2 indicates genetic disease-related proteins in OMIM, while the red color indicates the proteins identified by a literature search.

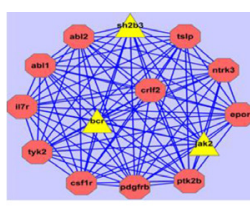

Complex 1 relation score $=6.5$ 14 nodes 91 edges

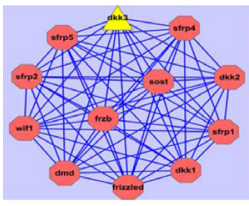

Complex 3 relation score $=5.5$ 12 nodes 66 edges

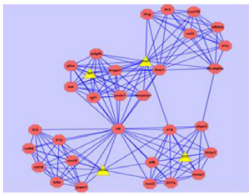

Complex 5 relation

score $=4.912$

34 nodes

167 edges
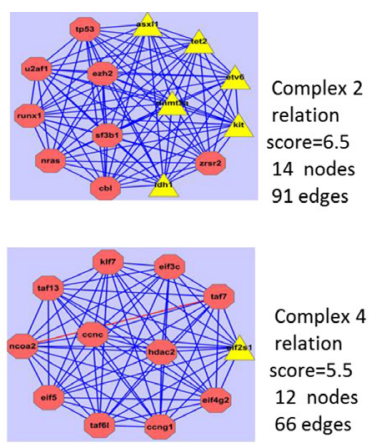

Complex 4 relation score $=5.5$

12 nodes 66 edges

Figure 2. Molecular complexes obtained by MCOMD algorithm analysis. 


\section{GO analysis of gene/protein in the molecular complexes}

The GO database was used for gene annotation and function-enrichment analysis of the 5 molecular complexes. The PV-related target genes were separately enriched in various biological processes, including peptide tyrosinase acidification, cell metabolism, macromolecular biosynthesis, gene expression, Wnt signal pathway regulation, and immune regulation. The major molecular functions of these genes included kinase activity, receptor binding, and cytokine activity, while the cell components were mainly concentrated in the nucleus, intracellular membranebound organelles, and the extracellular region. The five lowest enriched genes and protein in each molecular complex were chosen; these were concentrated in the nucleo-cytoplasm, cell membrane, extracellular space, cell surface membrane raft, and cell components, and regulated molecular functions such as nucleic acid binding, cytokine activity, protein activity, enzyme activity, and growth factor activity regulation (Tables 1-3). The biological process, molecular function, and cellular components of all molecular complexes are shown in Figures 3-5. The node size represented the number of genes, and the depth of color indicated the $\mathrm{P}$ value.

Table 1. Gene ontology (GO) analysis of biological processes related to the identified molecular complexes.

\begin{tabular}{|c|c|c|c|c|}
\hline \multirow{2}{*}{\multicolumn{5}{|c|}{ Complex 1}} \\
\hline & & & & \\
\hline & Regulation of long-term neuronal synaptic plasticity & $1.6730 \mathrm{E}-4$ & NRAS, KIT & $20 / 14305,0.1 \%$ \\
\hline 8542 & Visual learning & $2.4249 \mathrm{E}-4$ & NRAS, KIT & $24 / 14305,0.1 \%$ \\
\hline 7632 & Visual behavior & $3.0786 \mathrm{E}-4$ & NRAS, KIT & $27 / 14305,0.1 \%$ \\
\hline 9416 & Response to light stimulus & $3.3523 \mathrm{E}-4$ & NRAS, TP53, KIT & $144 / 14305,1.0 \%$ \\
\hline \multirow{2}{*}{\multicolumn{5}{|c|}{ Pentidyltyrosine phosnoolation }} \\
\hline & & & & \\
\hline 18212 & Peptidyl-tyrosine modification & $3.4494 \mathrm{E}-13$ & TYK2, PTK2B, PL & $41 / 14306,0.2 \%$ \\
\hline 6468 & Protein amino acid phosphorylation & $1.6742 \mathrm{E}-10$ & TYK2, NTRK3, BCR, PTK2B, PDGFRB, JAK2, ABL & $657 / 14306.4 .5 \%$ \\
\hline & Phosphorylation & $8.2677 \mathrm{E}-10$ & TYK2, NTRK3, BCR, PTK2B, PDGFRB, JAK2, A & $786 / 14306,5.4 \%$ \\
\hline 18193 & Peptidyl-amino aci & $1.4434 \mathrm{E}-9$ & TYK2, PTK2B, PDGFRB, JAK2, ABL1, ABL2 & $158 / 14306,1.1 \%$ \\
\hline \multicolumn{5}{|c|}{ 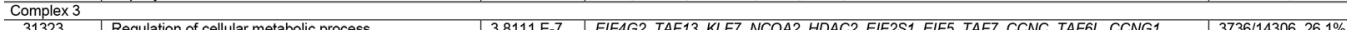 } \\
\hline $\begin{array}{l}31323 \\
19222 \\
\end{array}$ & $\begin{array}{l}\text { Regulation of cellular metabolic process } \\
\text { Regulation of metabolic process }\end{array}$ & \begin{tabular}{|l|l|}
$3.8111 \mathrm{E}-7$ \\
$6.4535 \mathrm{E}-7$
\end{tabular} & \begin{tabular}{|l} 
EIF4G2, TAF13, KLF7, NCOA2, HDAC2, EIF2S1, EIF5, TAF7, CCNC, TAFGL, CCNG1 \\
$E I F 4 G 2$, TAF13, KLF7, NCOA2, HDAC2, EIF2S1, EIF5, TAF, CCNC, TAFGL CCNG1
\end{tabular} & \begin{tabular}{|l|l|}
$3736 / 14306.26 .1 \%$ \\
$3919 / 14306.27 .3 \%$ \\
\end{tabular} \\
\hline 10556 & Regulation of macromolecule biosynthetic process & $9.6108 \mathrm{E}-7$ & EIF4G2, TAF13, KLF7, NCOA2, HDAC2, EIF2S1, EIF5, TAFT, CCNC, TAFGL & $2877 / 14306.20 .1 \%$ \\
\hline 10468 & Regulation of gene expression & $1.1415 \mathrm{E}-6$ & EIF4G2, TAF13, KLF7, NCOA2, HDAC2, EIF2S1, EIF5, TAF7, CCNC, TAFGL & $2928 / 14306,20.4 \%$ \\
\hline 31326 & Regulation of cellular biosynthetic process & $1.5499 \mathrm{E}-6$ & EIF4G2, TAF13, KLFT, NCOA2, HDAC2, EIF2S1, EIF5, TAFT, CCNC, TAF6L & $3021 / 14306,21.1 \%$ \\
\hline \multirow{2}{*}{\multicolumn{5}{|c|}{ Negative regulation of Wnt receptor signaling pathway }} \\
\hline & Negative regulation of Wnt receptor signaling pathway & $9.8887 \mathrm{E}-13$ & DKK2, DKK3, DKK & $54 / 14306,0.3 \%$ \\
\hline 30111 & Regulation of Wnt receptor signaling pathway & $1.9144 \mathrm{E}-11$ & DKK2, DKK 3 , DKK1, SFRP1, SFRP2, FRZB & $87 / 14306,0.6 \%$ \\
\hline 35467 & Negative regulation of signaling pathway & $1.9780 \mathrm{E}-10$ & DKK2, DKK, DKK1, SOST, SFRP1, SFRP2, FRZB & $262 / 14306,1.8 \%$ \\
\hline 16055 & Wht receptor signaling pathway & $2.2316 \mathrm{E}-10$ & SFRP5, SOST, SFRP1, SFRP2, SFRP4, WIF1 & $130 / 14306,0.9 \%$ \\
\hline 10648 & Negative regulation of cell communication & $9.5324 \mathrm{E}-10$ & DKK2, DKK3, DKK1, SOST, SFRP1, SFRP2, FRZB & $328 / 14306,2.2 \%$ \\
\hline \multicolumn{5}{|c|}{ TNE PTGS2 TLR2 NFKBIA TIR3 I10 CXCL10 PCSK1 CD86 TNFA IFNB1 } \\
\hline 9617 & Response to bacterium & $4.4647 \mathrm{E}-23$ & $\begin{array}{l}\text { TNF, PTGS2, TLR2, NFKBIA, TLR3, IL10, CXCL10, PCSK1, CD86, TNFA, IFNB1, } \\
\text { SERPINE1, IFNG, IL1B, NOS2 CD14, IL1A }\end{array}$ & $232 / 14305,1.6 \%$ \\
\hline 42127 & Regulation of cell proliferation & $9.6585 \mathrm{E}-21$ & $\begin{array}{l}\text { IFKBIA, IL10, TIMP1, IL11, CXCL10, CD86, } \\
\text { IG, IL1B, ANGPT11, NOS2, IL1A }\end{array}$ & $849 / 14305,5.9 \%$ \\
\hline 48518 & Positive regulation of biological process & $4.6294 \mathrm{E}-20$ & 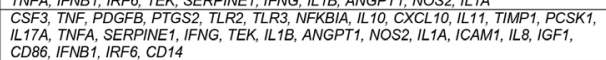 & $2209 / 14305,15.4 \%$ \\
\hline 51707 & Response to other organism & $7.8380 \mathrm{E}-20$ & $\begin{array}{l}\text { TNF, , PTGS2, TLR2, NFKBIA, TLR3, IL10, CXCL10, PCSK1, CD86, TNFA, IFNB1, } \\
\text { SERINE1, IFNG. IL1B, NOS2, CD14, IL1A }\end{array}$ & $358 / 14305,2.5 \%$ \\
\hline 2376 & Immune system process & $1.0806 \mathrm{E}-19$ & $\begin{array}{l}\text { CSF3, ICAM1, TNF, IL8, PDGFB, CXCL2, TLR2, NFKBIA, TLR3, IL10, TIMP1, IL11, } \\
\text { CXCL10, CD86, IL17A, TNFA, IFNB1, IFNG, IL1B, NOS2, CD14, IL1A }\end{array}$ & $949 / 14305,6.6 \%$ \\
\hline
\end{tabular}

Only the first 5 molecular function categories of enrichment analysis listed. "Total percentage of number of target genes in function categories with respect to the functional annotation of target gene.

\section{Molecular complex pathway enrichment}

The proteins included in the 5 molecular complexes were submitted online in order to identify the relevant pathways; these proteins were seen to mainly regulate the JAK-STAT signal transduction pathway, neurotrophic factor signaling pathway, Wnt signaling pathway, Toll-like receptor signal transduction pathway, and RIG-I-like receptor signal transduction pathway, as well as chronic myeloid leukemia, cancer, and acute myeloid leukemia (Table 4). 
Table 2. Gene ontology (GO) analysis of molecular functions of the identified molecular complexes.

\begin{tabular}{|c|c|c|c|c|}
\hline$\overline{G O-I D}$ & Description & Pvalue & Genes & Total frequency $y^{\prime \prime}$ \\
\hline \multicolumn{5}{|c|}{ Complex 1} \\
\hline 3682 & Chromatin binding & $4.5153 \mathrm{E}-4$ & SF3B1, DNMT3A, TP53 & $172 / 15443,1.1 \%$ \\
\hline 3676 & Nucleic acid binding & $5.6971 \mathrm{E}-4$ & SF3B1, DNMT3A, CBL, EZH2, U2AF1, TP53, ZRSR2, ETV6, RUNX1 & $\begin{array}{l}3252 / 15443, \\
21.0 \%\end{array}$ \\
\hline 5020 & Stem cell factor receptor activity & $9.0656 \mathrm{E}-4$ & & \\
\hline 51718 & DNA (cytosine-5-)-methyltransferase activity, acting on CpG substrates & $1.8124 \mathrm{E}-3$ & DNMT3A & $2 / 15443,0.0 \%$ \\
\hline 35033 & Histone deacetylase regulator activity & $1.8124 \mathrm{E}-3$ & TP53 & $2 / 15443,0.0 \%$ \\
\hline \multicolumn{5}{|c|}{ Histone deacerylase regulator activity } \\
\hline 4713 & Protein tyrosine kinase activity & $1.0082 \mathrm{E}-13$ & TYK2, NTRK3, PTK2B, PDGFRB, JAK2, ABL1, ABL2, CSF1R & $139 / 1544,40.9 \%$ \\
\hline 4715 & Non-membrane spanning protein tyrosine kinase activity & $1.7700 \mathrm{E}-10$ & TYK2, PTK2B, JAK2, ABL1, ABL2 & $40 / 15444,0.2 \%$ \\
\hline 4672 & Protein kinase activity & $1.9642 \mathrm{E}-10$ & TYK2, NTRK3, BCR, PTK2B, PDGFRB, JAK2, ABL1, ABL2, CSF1R & $568 / 1544,43.6 \%$ \\
\hline 16773 & Phosphotransferase activity, alcohol group as acceptor & $9.8214 \mathrm{E}-10$ & TYK2, NTRK3, BCR, PTK2B, PDGFRB, JAK2, ABL1, ABL2, CSF1R & $681 / 1544,44.4 \%$ \\
\hline 16301 & Kinase activity & $2.0710 \mathrm{E}-9$ & TYK2, NTRK3, BCR, PTK2B, PDGFRB, JAK2, ABL1, ABL2, CSF1R & $741 / 1544,44.7 \%$ \\
\hline \multicolumn{5}{|c|}{ 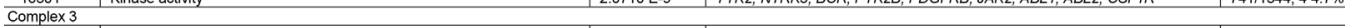 } \\
\hline 3743 & Translation initiation factor activity & $2.6179 \mathrm{E}-6$ & EIF4G2, EIF2S1, EIF5 & $40 / 15444,0.2 \%$ \\
\hline 16563 & Transcription activator activity & $6.5016 \mathrm{E}-6$ & KLF7, NCOA2, HDAC2, TAF7, TAFGL & $429 / 1544,42.7 \%$ \\
\hline 3713 & Transcription coactivator activity & $\begin{array}{l}0.010 \mathrm{E}-0 \\
1.1164 \mathrm{E}-5\end{array}$ & $\begin{array}{l}\text { KLFT, NCOA2, TAF?, TAFGL } \\
\end{array}$ & $\frac{425 / 154,42,470}{215 / 154,41.3 \%}$ \\
\hline 8135 & Translation factor activity, nucleic acid binding & $1.6270 \mathrm{E}-5$ & EIF4G2, EIF2S1, EIF5 & $73 / 15444,0.4 \%$ \\
\hline 8134 & Transcription factor binding & $1.6886 \mathrm{E}-5$ & KLF7, NCOA2, HDAC2, TAF7, TAFGL & $522 / 1544,43.4 \%$ \\
\hline \multicolumn{5}{|c|}{ KLFI, NCOAZ, HDAC2, IAFI, IAFGL } \\
\hline 17147 & Wnt-protein binding & $1.2056 \mathrm{E}-10$ & SFRP1, SFRP2, SFRP4, FRZB & $15 / 15444,0.0 \%$ \\
\hline 2162 & Dystroglycan binding & $1.9414 \mathrm{E}-3$ & DMD & $3 / 15444,0.0 \%$ \\
\hline 0998 & Nitric-oxide synthase binding & $3.2337 \mathrm{E}-3$ & DMD & $5 / 15444,0.0 \%$ \\
\hline \multirow{2}{*}{\multicolumn{5}{|c|}{$\frac{5109}{\text { Complex } 5}$}} \\
\hline & & & & \\
\hline 5125 & Cytokine activity & $2.8668 \mathrm{E}-17$ & $\begin{array}{l}\text { CSF3, IL17A, TNFA, TNF, IL8, IFNB1, IFNG, CXCL2, IL1B, IL1A, IL10, } \\
\text { CXCL10, IL11 }\end{array}$ & $198 / 15443,1.2 \%$ \\
\hline 5126 & Cytokine receptor binding & $8.0428 \mathrm{E}-16$ & $\begin{array}{l}\text { CSFF, TNFA, TNF, IL8, IFNB1, IFNG, CXCL2, IL1B, IL1A, IL10, CXCL10, } \\
\text {, }\end{array}$ & $187 / 15443,1.2 \%$ \\
\hline 5102 & Receptor binding & $1.6455 \mathrm{E}-13$ & $\begin{array}{l}\text { CS11 } \\
\text { CSF3, ICAM1, TNF, IL8, PDGFB, CXCL2, IGF1, IL10, IL11, CXCL10, IL17A, } \\
\text { TNFA, IFNB1, IFNG, IL1B, ANGPT1, IL1A }\end{array}$ & $923 / 15443,5.9 \%$ \\
\hline & tor binding & $1.2325 \mathrm{E}-8$ & CSF3, PDGFB, IL1B, IL1A, IL10, IL11 & $82 / 15443,0.5 \%$ \\
\hline 80851 & $\begin{array}{l}\text { Grownt ractor recepetor binaing } \\
\text { Growth factor activity }\end{array}$ & $\begin{array}{l}\frac{1.32325 \mathrm{E}-8}{6.6913 \mathrm{E}-7} \\
-19\end{array}$ & 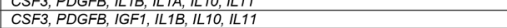 & $\begin{array}{l}8 / 260 / 154443,0.5 \% \\
1.0 \% \\
\end{array}$ \\
\hline
\end{tabular}

List only the first 5 molecular function categories of enrichment analysis results. ${ }^{\text {TT}}$ Total percentage of the number of target gene in the current functional categories to the functional annotation of target gene.

Table 3. Gene ontology (GO) analysis of cellular components of the identified molecular complexes.

\begin{tabular}{|c|c|c|c|c|}
\hline GO-ID & Description & $P$ value & Genes & Total frequency" \\
\hline \multicolumn{5}{|c|}{ 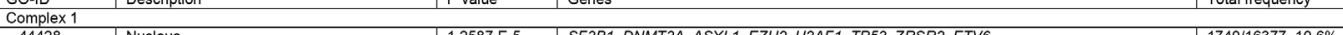 } \\
\hline 44428 & Nucleus & $1.2587 \mathrm{E}-5$ & SF3B1, DNMT3A, ASXL1, EZH2, U2AF1, TP53, ZRSR2, ETV6 & $1740 / 16377,10.6 \%$ \\
\hline 31519 & PcG protein complex & $7.8578 \mathrm{E}-5$ & ASXL1, EZH2 & $17 / 16377,0.1 \%$ \\
\hline 5681 & Spliceosomal complex & $1.4427 \mathrm{E}-4$ & SF3B1, U2AF1, ZRSR2 & $134 / 16377,0.8 \%$ \\
\hline 43231 & Intracellular membrane-bounded organelle & $1.5615 \mathrm{E}-4$ & DNMT3A, EZH2, ASXL1, CBL, TP53, KIT, SF3B1, NRAS, U2AF1, IDH1, ZRSR2, ETV6, & $8348 / 16377,50.9 \%$ \\
\hline 43227 & Membrane-bounded organelle & $1.5786 \mathrm{E}-4$ & $\begin{array}{l}\text { DNMT3A, EZH2, ASXL1, CBL, TP53, KIT, SF3B1, NRAS, U2AF1, IDH1, ZRSR2, ETV6, } \\
\text { RUNX1 }\end{array}$ & $8355 / 16377,51.0 \%$ \\
\hline \multicolumn{5}{|c|}{ Complex 2} \\
\hline \multirow{2}{*}{\multicolumn{5}{|c|}{ F }} \\
\hline & & & & \\
\hline 5669 & Transcription factor TFIID complex & $6.9693 \mathrm{E}-5$ & TAF13, TAF7 & $\begin{array}{l}2 / 11, \\
18.1 \% \\
\end{array}$ \\
\hline 70461 & SA GA-type complex & $1.0296 \mathrm{E}-4$ & TAF7, TAFGL & $\begin{array}{l}2 / 11, \\
18.1 \%\end{array}$ \\
\hline 118 & Histone deacetylase complex & $2.5517 \mathrm{E}-4$ & HDAC2, TAFGL & $\begin{array}{l}2 / 11, \\
18.1 \%\end{array}$ \\
\hline 44451 & Nucleoplasm part & $4.3225 \mathrm{E}-4$ & TAF13, HDAC2, TAF7, TAFGL & $\begin{array}{l}4 / 11 \\
36.3 \%\end{array}$ \\
\hline 123 & Histone acetyltransferase complex & $4.9362 \mathrm{E}-4$ & TAF7, TAFGL & $\begin{array}{l}2 / 11 \\
18.1 \%\end{array}$ \\
\hline \multicolumn{5}{|c|}{ Complex 4} \\
\hline 5576 & Extracellular region & $8.0772 \mathrm{E}-9$ & SFRP5, DKK2, DKK 3 , DKK1, SOST, SFRP1, SFRP2, SFRP4, WIF1, FRZB & $2027 / 16378,12.3 \%$ \\
\hline 5615 & Extra & $1.1225 \mathrm{E}-7$ & & $747 / 16378,4.5 \%$ \\
\hline 44421 & Extracellular r & $7.4280 \mathrm{E}-7$ & SFRP5, DKK2, DKK3, SOST, SFRP1, SFRP4, FRZB & $985 / 16378,6.0 \%$ \\
\hline \multicolumn{5}{|l|}{ Complex 5} \\
\hline 44421 & Extracellular region part & $4.7822 \mathrm{E}-19$ & $\begin{array}{l}\text { CSF3, ICAM1, TNF, IL8, CXCL2, IGF1, MMP1, IL10, TIMP1, IL11, CXCL10, PCSK1, IL17A, } \\
\text { TNFA. IFNB1, IFNG SERPINE1, IL1B, ANGPT1, CD14, ILAA }\end{array}$ & $986 / 16378,6.0 \%$ \\
\hline 5615 & Extracellular space & $2.3005 \mathrm{E}-18$ & $\begin{array}{l}\text { CSF3, ICAM1, TNF, IL8, CXCL2, IGF1, IL10, IL11, CXCL10, PCSK1, IL17A, TNFA, IFNB1, } \\
\text { SERPINE1, IFNG, LLB, ANGPT1, CD14, LL1A }\end{array}$ & $748 / 16378,4.5 \%$ \\
\hline 5576 & Extracellular region & $6.5374 \mathrm{E}-14$ & $\begin{array}{l}\text { CSF3, IIAM1, TNF, IL8, PDGFB, CXCL2, IGF1, MMP1, IL10, TIMP1, IL11, CXCL10, } \\
\text { PCSK1, LL17A, TNFA, IFNB1, IFNG, SERPINE1, IL1B, ANGPT1, CD14, IL1A }\end{array}$ & $2028 / 16378,12.3 \%$ \\
\hline 9986 & Cell surt & 1.68 & ICAM1, LD86, TNFA, TNF, , DDGFB, TEK, TLR2, IL1A & $2.0 \%$ \\
\hline 45121 & Membrane raft & $3.1278 \mathrm{E}-7$ & TNFA, TNF, PTGS2, TEK, ANGPT1, CD14 & $149 / 16378,0.9 \%$ \\
\hline
\end{tabular}

List only the first 5 molecular function categories of enrichment analysis results. "Total percentage of the number of target gene in the current functional categories to the functional annotation of target gene. 

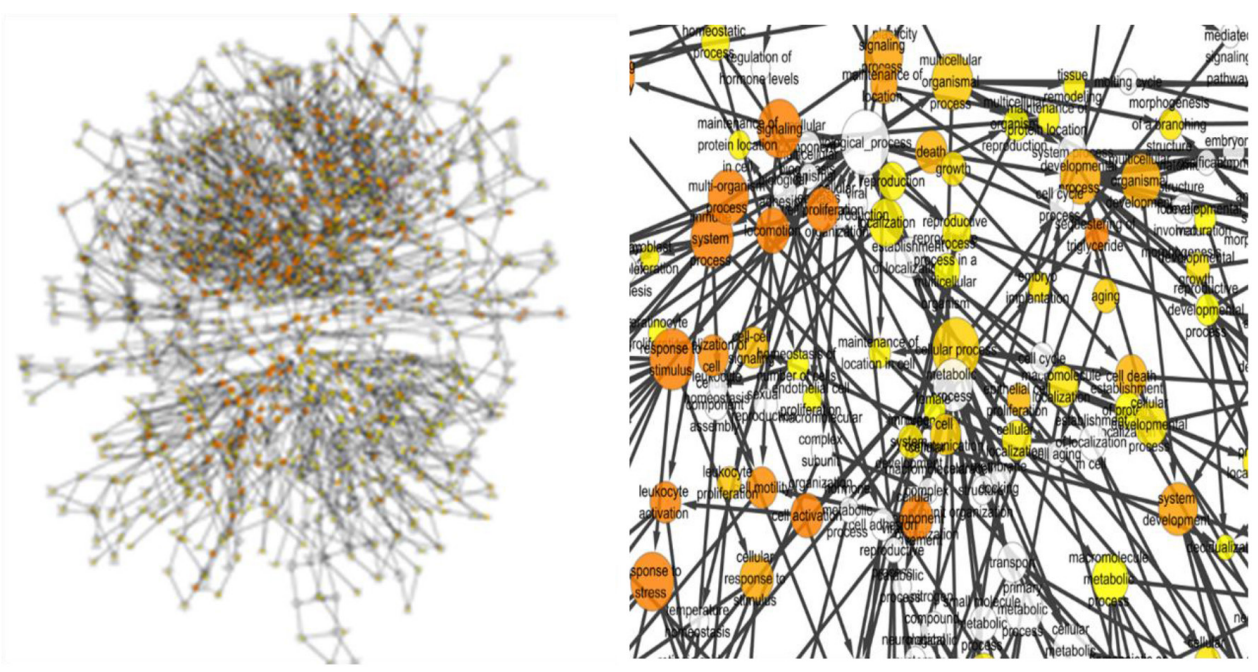

Figure 3. Biological processes of complex 5 (the whole and the part).

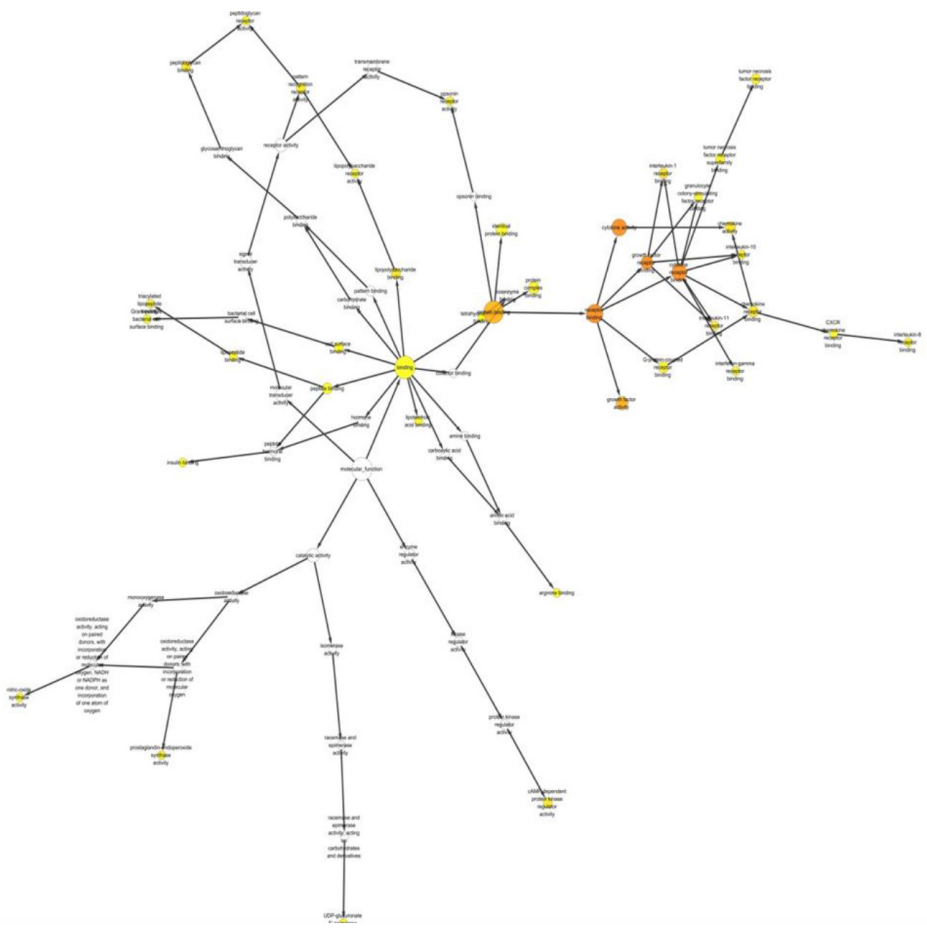

Figure 4. Molecular function of complex 5 . 


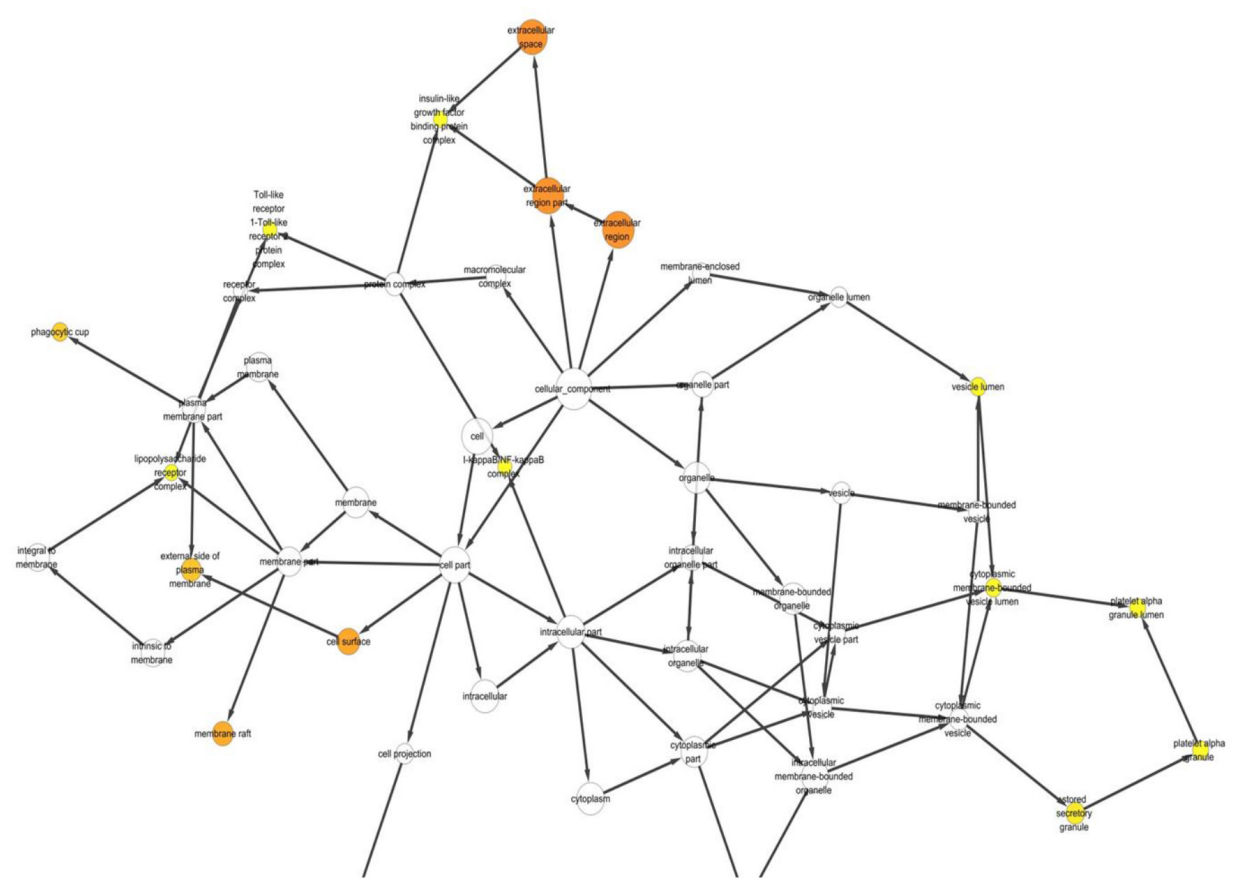

Figure 5. Cell components of complex 5.

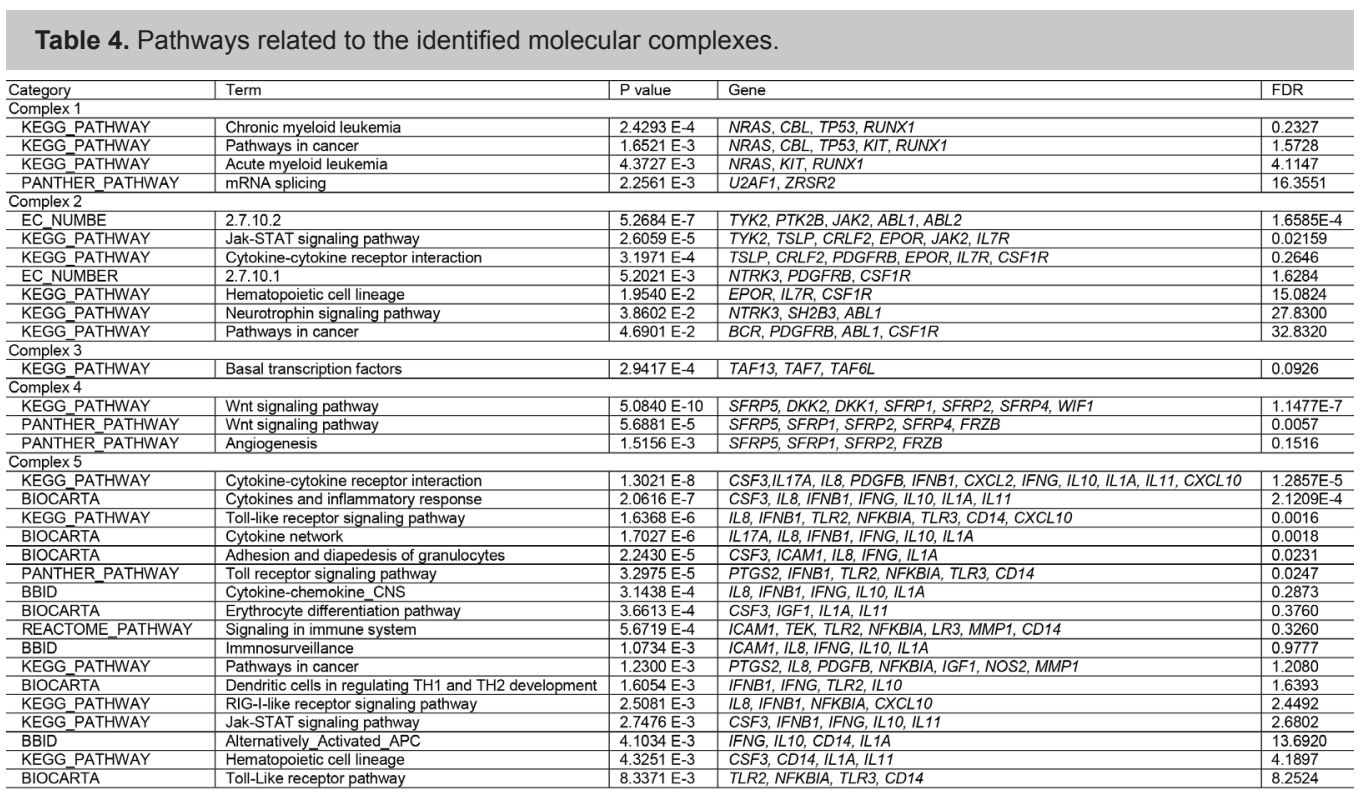

Genetics and Molecular Research 15 (1): gmr.15017169 


\section{DISCUSSION}

The major purpose of a bioinformatic analysis of molecular regulation networks is to elucidate the temporo-spatial mechanism of molecular regulation using a model. Identification of the precise molecular mechanism and complicated regulation of gene expression could be useful in establishing various network models to describe the behavior of biological systems. Gene expression is dependent on its interaction with other genes and regulation. Elucidation of the relationship between diseases, genes, and drugs using a network plays a significant role in the design of drugs.

A molecular regulation network construct is based on the foundation of the formation and development of the internet for the study of a network dynamic model. The most common network evolution models of this day include the rule network model, random network model, small-world network model, and scale-free network model. In general, complicated molecular regulation networks display 4 properties: degree distribution, the shortest path, aggregation, and robustness. The study of the systemic network properties of living organisms is at a preliminary stage; however, organismal networks are based on some general universal rules. Ravasz et al. (2002) split an algorithm-based metabolic network into smaller modular sub-networks, and discovered that the majority of the reactions within the same module belonged to the same metabolic pathways. This indicated that each sub-module has relatively independent biological functions; therefore, these were denoted as biological function modules. Based on these results, a compilation of genes recovered from OMIM were combined with the results of literature mining to build a network of protein-protein interactions in PV in this study. Although the extracted experimental data were related to genes, the identified literature proved its function and interaction with other molecules at a protein level. Therefore, the network set up via literature mining could illuminate protein-protein interactions in molecular complexes.

A PV protein-protein interaction network was built using the 54 genes identified from OMIM; this network included 541 nodes (proteins) and 1771 sides (mutual effects). Because of the enormity of the internet, the MCOMD algorithm was used to estimate the network area, through a correlation integral. Correlation integrals illustrate the correlation of proteins at a specific area. Proteins in these complexes that regulated known biological processes and pathways, and with known molecular functions and cellular components were excluded to allow for the identification of the function of unknown genes or novel molecular functions (Bader and Hogue, 2003; Zhou et al., 2014). Five molecular compounds with correlation integral $>4$ were identified in this study.

Gene ontology is a new perspective in the study of functional genomics. GO develops effective bioinformatic methods to realize the targets of genetic network analysis and the functional prediction of unknown genes using previously existing biological architecture and knowledge. GO analyzes for three stand-alone systems: cellular component, biological process, and molecular function. Based on these, GO analysis identified 5 compounds that were considerably enriched, and the characteristics of classification were determined by regional GO. DAVID enriches the biological information of single genes in addition to annotating large genes from different species.

Compound 2 was found to regulate several biological processes, including the phosphorylation of the peptide tyrosine and the amino acids in proteins; the molecular function of compound 2 included the regulation of tyrosine kinase and phosphatase activity, while its cellular component was concentrated in the plasma membrane. Studies conducted since 2005 revealed that the morbidity of PV was closely related to the JAK gene mutation. JAK, a tyrosine phosphatase 
protein family expressed in mammals, is composed of four members: JAK1, JAK2, JAK3, and TYK2. Tyrosine phosphatase, which suppresses cytokine signaling and inhibits activated STATs, negatively regulates the JAK/STAT pathway (Rawlings et al., 2004; Wang et al., 2009).

Phosphorylation of the tyrosyl residue is the key regulatory mechanism in the eukaryotic signaling pathway, and is regulated by PTKs and PTPs. PTP de-phosphorylation and PTK phosphorylation are in dynamic equilibrium, and are closely related to cellular proliferation, differentiation, signal transduction, and regulation of cell cycle. Some studies have reported that this balance can be disrupted by up-regulating PTK expression; this could lead to the accumulation of proteins related to tyrosine phosphorylation in the cell, which in turn causes abnormal cell proliferation and differentiation, as well as disease. This imbalance could also be caused by the decline in PTP activity as a result of gene mutation, leading to an increase in GAP-associated proteins (affecting tyrosine phosphorylation) in the cell. The increase in intracellular protein phosphorylation could be a key to unlocking the cell proliferation and differentiation mechanism (Weerkamp et al., 2006).

The gene/protein interaction in molecule 4 is responsible for various biological processes (regulation of Wnt signal pathway) and molecular functions (combining Wnt protein with nitric oxide synthase), while its cellular component is located in the extracellular space and region. The classical Wnt pathway has been extensively researched in malignant diseases of the blood. Weerkamp et al. (2006) and Khan et al. (2007) reported that ALL patients expressed abnormalities in the Wnt pathway; additionally, many genes and receptors belonging to the Wnt family have been identified in precursor B-cell lymphoblastic leukemic cells. Wnt3 stimulation leads to $\beta$-catenin accumulation, rapid cell proliferation, and a discernible change in cell cycle and apoptosis-related gene function. Therefore, abnormalities in the classical Wnt pathway is a potential pathogenesis of malignant disease (Valencia et al., 2009). A previous study on AML reported that the genes encoding the Wnt pathway antagonist proteins DKK1 and DKK3 undergo methylation in vitro (cell line and specimen) (Wu et al., 2009). B-CLL patients highly express Wnt proteins and their homologous receptor genes. The study of B-CLL and its development in an animal (mouse) model revealed that an increase in impaired Fz-6 and $\beta$-catenin expression led to a decrease in disease progression (Ashihara et al., 2009). $\beta$-catenin is highly expressed in myeloma cell lines; cell proliferation is disrupted upon deletion of the $\beta$-catenin small interfering RNA (Riley et al., 2008).

The biological process of compound 1 is similar to that of 3 and 5 , and includes the regulation of cellular metabolism, macromolecular biosynthesis, regulation of gene expression, cell proliferation, and immune regulation. The molecular function of compound 1 includes nucleic acid clustering, cytokine activity, enzymatic activity, and transcription factor clustering. Compound 1 is mainly located in the nucleus, intracellular membrane-bound organelle, nucleoplasm, and the extracellular space. TP53 (coding for p53) is an important tumor suppressor gene, which retards the cell cycle, restrains cell proliferation, induces cell differentiation, activates cell aging and apoptosis, participates in DNA damage and repair (maintain genomic balance), regulates the energy metabolism, and restrains tumor angiogenesis, via transcriptional regulation of many key genes such as p21, Bax, and Puma when the cell is stimulated by carcinogenic factors, thereby preventing the genesis and development of tumor (Teodoro et al., 2006; Riley et al., 2008; Feng and Levine, 2010; Feng et al., 2011; Kumar et al., 2011). More than 50\% tumors are estimated to express mutated TP53, which could lead to an abnormal p53 signal transduction pathway (Xiong et al., 2008; Thol et al., 2010; Kumar et al., 2011). Interferon $\alpha$ (IFN $\alpha$ ) up-regulates the activity of the NK gene via the STATE1 and STAT3 signal pathways. Isocitrate dehydrogenase catalyzes the 
conversion of isocitric acid to $\alpha$-oxoglutarates, such as IDHA, IDH2, and IDH3. IDH1/2 mutations can undergo one of two mechanisms resulting in tumor formation: 1) decline in the ability of isocitric acid to introduce $\alpha-K G$, leading to the accumulation of hypoxia-inducible factor 1a (HIF1a), and influencing the formation, transference, and resistance of tumor vessels (Rakheja et al., 2011); 2) development of new enzymatic ability by the mutated IDH1/2, leading to the accumulation of D-2$H G$ and subsequent competitive inhibition of $\alpha-K G$-dependent dioxygenase, which leads to the abnormal expression of a series of signal conductors, thereby influencing transcription regulation, angiogenesis, differentiation, proliferation, anti-apoptosis activity, transference, and gene invasion (Ward et al., 2010; Frezza et al., 2010; Andersson et al., 2011; Westman et al., 2013).

The biological pathways regulated by the various molecular complexes were separately enriched in our study: compound 1 regulated the Jak-STAT signaling pathway, neurotrophin signaling pathway, Wnt signaling pathway, Toll-like receptor signaling pathway, Toll receptor signaling pathway, RIG-I-like receptor signaling pathway, and erythrocyte differentiation pathway; compound 2 was associated with cancer pathways that were associated with different types of cancer, including chronic myeloid leukemia and acute myeloid leukemia; compound 3 regulated complex biological behaviors, including that of hematopoietic cell lineage, immune function, signal transcription, angiogenesis, cytokine activation and function, and inflammatory response. Previous studies have indicated that myeloproliferative diseases are highly sensitive to cytokines, and the genes initiating and regulating the related signal pathways could offer a means for the molecular treatment and cure of PV. The identified compounds are representative of the complicated biological behavior of PV, and its extensive correlation with other tumors; additionally, these compounds describe $\mathrm{PV}$ as a network system composed of various signal pathways and genes. Therefore, $\mathrm{PV}$ can be considered as a complicated disease requiring coordinated regulation of multiple genes and signal pathways (and not caused by any individual gene or signal pathway). However, this network could contain a specific "target spot" to facilitate its treatment. The genes and signal pathways identified in this experiment should offer a reliable direction for further studies into, and treatment of, PV. However, our results must be further verified in the lab.

\section{Conflicts of interest}

The authors declare no conflict of interest.

\section{REFERENCES}

Amberger J, Bocchini CA, Scott AF and Hamosh A (2009). McKusick's Online Mendelian Inheritance in Man (OMIM). Nucleic Acids Res. 37: D793-D796. http://dx.doi.org/10.1093/nar/gkn665

Andersson AK, Miller DW, Lynch JA, Lemoff AS, et al. (2011). IDH1 and IDH2 mutations in pediatric acute leukemia. Leukemia 25: 1570-1577. http://dx.doi.org/10.1038/leu.2011.133

Ashihara E, Kawata E, Nakagawa Y, Shimazaski C, et al. (2009). beta-catenin small interfering RNA successfully suppressed progression of multiple myeloma in a mouse model. Clin. Cancer Res. 15: 2731-2738. http://dx.doi.org/10.1158/10780432.CCR-08-1350

Bader GD and Hogue CW (2003). An automated method for finding molecular complexes in large protein interaction networks. BMC Bioinformatics 4: 2. http://dx.doi.org/10.1186/1471-2105-4-2

Burkard TR, Rix U, Breitwieser FP, Superti-Furga G, et al. (2010). A computational approach to analyze the mechanism of action of the kinase inhibitor bafetinib. PLOS Comput. Biol. 6: e1001001. http://dx.doi.org/10.1371/journal.pcbi.1001001

Dennis G, Jr., Sherman BT, Hosack DA, Yang J, et al. (2003). DAVID: Database for Annotation, Visualization, and Integrated Discovery. Genome Biol. 4: 3. http://dx.doi.org/10.1186/gb-2003-4-5-p3

Feng $Z$ and Levine AJ (2010). The regulation of energy metabolism and the IGF-1/mTOR pathways by the p53 protein. Trends 
Cell Biol. 20: 427-434. http://dx.doi.org/10.1016/j.tcb.2010.03.004

Feng Z, Zhang C, Wu R and Hu W (2011). Tumor suppressor p53 meets microRNAs. J. Mol. Cell Biol. 3: 44-50. http://dx.doi. org/10.1093/jmcb/mig040

Frezza C, Tennant DA and Gottlieb E (2010). IDH1 mutations in gliomas: when an enzyme loses its grip. Cancer Cell 17: 7-9 http://apps.cytoscape.org/apps/bingo. http://dx.doi.org/10.1016/j.ccr.2009.12.031

Huang W, Sherman BT and Lempicki RA (2009). Systematic and integrative analysis of large gene lists using DAVID bioinformatics resources. Nat. Protoc. 4: 44-57. http://dx.doi.org/10.1038/nprot.2008.211

Khan NI, Bradstock KF and Bendall LJ (2007). Activation of Wnt/beta-catenin pathway mediates growth and survival in B-cell progenitor acute lymphoblastic leukaemia. Br. J. Haematol. 138: 338-348. http://dx.doi.org/10.1111/j.13652141.2007.06667.x

Kralovics R, Guan Y and Prchal JT (2002). Acquired uniparental disomy of chromosome 9p is a frequent stem cell defect in polycythemia vera. Exp. Hematol. 30: 229-236. http://dx.doi.org/10.1016/S0301-472X(01)00789-5

Kumar M, Lu Z, Takwi AA, Chen W, et al. (2011). Negative regulation of the tumor suppressor p53 gene by microRNAs. Oncogene 30: 843-853. http://dx.doi.org/10.1038/onc.2010.457

Levine RL and Gilliland DG (2008). Myeloproliferative disorders. Blood 112: 2190-2198. http://dx.doi.org/10.1182/ blood-2008-03-077966

Rakheja D, Konoplev S, Su M, Wheeler D, et al. (2011). High incidence of IDH mutations in acute myeloid leukaemia with cuplike nuclei. Br. J. Haematol. 155: 125-128. http://dx.doi.org/10.1111/j.1365-2141.2011.08646.x

Ravasz E, Somera AL, Mongru DA, Oltvai ZN, et al. (2002). Hierarchical organization of modularity in metabolic networks. Science 297: 1551-1555. http://dx.doi.org/10.1126/science.1073374

Rawlings JS, Rosler KM and Harrison DA (2004). The JAK/STAT signaling pathway. J. Cell Sci. 117: 1281-1283. http://dx.doi. org/10.1242/jcs.00963

Riley T, Sontag E, Chen P and Levine A (2008). Transcriptional control of human p53-regulated genes. Nat. Rev. Mol. Cell Biol. 9: 402-412. http://dx.doi.org/10.1038/nrm2395

Shannon P, Markiel A, Ozier O, Baliga NS, et al. (2003). Cytoscape: a software environment for integrated models of biomolecular interaction networks. Genome Res. 13: 2498-2504. http://dx.doi.org/10.1101/gr.1239303

Teodoro JG, Parker AE, Zhu X and Green MR (2006). p53-mediated inhibition of angiogenesis through up-regulation of a collagen prolyl hydroxylase. Science 313: 968-971. http://dx.doi.org/10.1126/science.1126391

Thol F, Weissinger EM, Krauter J, Wagner K, et al. (2010). IDH1 mutations in patients with myelodysplastic syndromes are associated with an unfavorable prognosis. Haematologica 95: 1668-1674. http://dx.doi.org/10.3324/haematol.2010.025494

Vailaya A, Bluvas P, Kincaid R, Kuchinsky A, et al. (2005). An architecture for biological information extraction and representation. Bioinformatics 21: 430-438. http://dx.doi.org/10.1093/bioinformatics/bti187

Valencia A, Román-Gómez J, Cervera J, Such E, et al. (2009). Wnt signaling pathway is epigenetically regulated by methylation of Wnt antagonists in acute myeloid leukemia. Leukemia 23: 1658-1666. http://dx.doi.org/10.1038/leu.2009.86

Wang Z, Jin H, Xu R, Mei Q, et al. (2009). Triptolide downregulates Rac1 and the JAK/STAT3 pathway and inhibits colitisrelated colon cancer progression. Exp. Mol. Med. 41: 717-727. http://dx.doi.org/10.3858/emm.2009.41.10.078

Ward PS, Patel J, Wise DR, Abdel-Wahab O, et al. (2010). The common feature of leukemia-associated IDH1 and IDH2 mutations is a neomorphic enzyme activity converting alpha-ketoglutarate to 2-hydroxyglutarate. Cancer Cell 17: 225234. http://dx.doi.org/10.1016/j.ccr.2010.01.020

Weerkamp F, van Dongen JJ and Staal FJ (2006). Notch and Wht signaling in T-lymphocyte development and acute lymphoblastic leukemia. Leukemia 20: 1197-1205. http://dx.doi.org/10.1038/sj.leu.2404255

Westman MK, Pedersen-Bjergaard J, Andersen MT and Andersen MK (2013). IDH1 and IDH2 mutations in therapy-related myelodysplastic syndrome and acute myeloid leukemia are associated with a normal karyotype and with $\operatorname{der}(1 ; 7)$ (q10;p10). Leukemia 27: 957-959. http://dx.doi.org/10.1038/leu.2012.347

Wu QL, Zierold C and Ranheim EA (2009). Dysregulation of Frizzled 6 is a critical component of B-cell leukemogenesis in a mouse model of chronic lymphocytic leukemia. Blood 113: 3031-3039. http://dx.doi.org/10.1182/blood-2008-06-163303

Xiong W, Wu X, Starnes S, Johnson SK, et al. (2008). An analysis of the clinical and biologic significance of TP53 loss and the identification of potential novel transcriptional targets of TP53 in multiple myeloma. Blood 112: 4235-4246. http://dx.doi. org/10.1182/blood-2007-10-119123

Zhou C, Teng WJ, Yang J, Hu ZB, et al. (2014). Construction of a protein-protein interaction network for chronic myelocytic leukemia and pathway prediction of molecular complexes. Asian Pac. J. Cancer Prev. 15: 5325-5330. http://dx.doi. org/10.7314/APJCP.2014.15.13.5325 